\title{
The line tension and the generalized Young equation: the choice of dividing surface
}

\author{
A.I. Rusanov ${ }^{\mathrm{a}, *}$, A.K. Shchekin ${ }^{\mathrm{b}}$, D.V. Tatyanenko ${ }^{\mathrm{b}}$ \\ a Mendeleev Center, St. Petersburg State University, St. Petersburg 199034, Russia \\ ${ }^{\mathrm{b}}$ Research Institute of Physics, St. Petersburg State University, Ulyanovskaya 1, Petrodvorets, St. Petersburg 198504, Russia
}

Received 29 January 2004; accepted 19 April 2004

Available online 25 September 2004

\begin{abstract}
Using Gibbs method of dividing surfaces, the condition of equilibrium of a sessile drop on a flat non-deformable solid substrate is investigated. The dependence of the line tension on the curvature radius of the dividing three-phase contact line is found. It has been derived a relationship between the partial derivative of the line tension with respect to the curvature radius of the three-phase contact line (which stands in the generalized Young equation) and the total derivative of the line tension with respect to the same radius along the equilibrium states. Various approximated formulas of the generalized Young equation used in the literature are analyzed.
\end{abstract}

(C) 2004 Elsevier B.V. All rights reserved.

PACS: $68.08 . \mathrm{Bc} ;$ 68.35.Md; 05.70.Np; 82.65.Dp

Keywords: Line tension; Contact angle; Generalized Young equation; Dividing surface

\section{Introduction}

A partly wetting liquid forms, on a surface, sessile drops with a certain contact angle whose value is determined by the thermodynamic (adhesive) surface tensions of three interfaces and by the curvature radius of the three-phase contact line. For small drops, the dependence of the contact angle on the contact line radius is known to be considerably influenced by the line tension at the three-phase boundary. The generalized Young equation describes this dependence. Various generally non-equivalent forms of this equation are met in the literature (see, e.g., [1-3]), whose experimental verification is problematic due to accuracy restrictions at the present. For a system including a solid substrate (phase $\gamma$ ) in contact with a liquid drop (phase $\alpha$ ) and with its equilibrium vapour (phase $\beta$ ), the Young equation in the most general form (accounting for gravity, for the slope and strain of the substrate

\footnotetext{
* Corresponding author. Tel.: +7 8125542877.

E-mail address: rusanov@AR1047.spb.edu (A.I. Rusanov).
}

and for the line tension dependence on the contact angle and on the radius of the three-phase contact line) is [1]:

$\sigma^{\alpha \beta} \cos \theta=\sigma^{\beta \gamma}-\sigma^{\alpha \gamma}-\left(\frac{\kappa}{r}+\frac{\partial \kappa}{\partial r}\right)|\cos \varphi|$.

Here, $\sigma$ is the thermodynamic surface tension, $\theta$ is the equilibrium contact angle, $\varphi$ is the angle between the substrate surface and the local principal plane of the three-phase contact line, $r$ is the radius of the three-phase contact line, $\kappa$ is the corresponding thermodynamic line tension (or the effective line tension in case of substrate deformation [1]); double superscripts mark the quantities related to the interfaces. Eq. (1) corresponds to the choice of the surface of tension as a dividing surface between phases $\alpha$ and $\beta$.

Since Eq. (1) was derived [1] from the equilibrium condition for the grand thermodynamic potential, the partial derivative of $\kappa$ with respect to the radius of the three-phase contact line is evidently taken at the constancy of the temperature, the chemical potentials and the contact angle. Eq. (1) was also generalized for rough and heterogeneous surfaces $[4,5]$. 
Other problems related to the line tension have been actively studied in last several decades. Among them, a generalized Neumann-Young equation was obtained [6], the concept of line of tension was introduced [7], the theories of line tension based on different approaches to the detailed description of the system were developed and several estimates of the value of the line tension were presented [8-12], the stability conditions were examined and the possibility of line tension to have positive or negative values was commented [13-15]. New experimental methods for measuring the line tension were described and different applications of the line tension concept to the theories of formation of thin films, flotation, heterogeneous nucleation were found. A comprehensive list of references on most mentioned topics is given in the recent review papers [12]. A review of earlier works on the line tension can be found in reference [15].

In this work, we investigate the line tension dependence on the radius of a dividing line which corresponds to the variation of the radius of the dividing surface between phases $\alpha$ and $\beta$ at a fixed physical state of the system. An analogous dependence appears for surface tension in the known approach developed by Kondo [16]. We shall consider the simple case of a pure liquid on a flat uniform substrate $(\varphi=0)$. The drop size is implied to be large enough to neglect overlapping the surface layers at the liquid/substrate and liquid/vapour boundaries in the central part of the drop. Note that overlapping these layers at drop perimeter determines the line tension and is of principal importance. We shall obtain the drop equilibrium conditions written for excess quantities corresponding not only to the surface of tension, but also to an arbitrary choice of a dividing surface. We shall also discuss the physical meaning of the derivative $\partial \kappa / \partial r$ and outline the way of its explicit finding by numerical calculation. This way is also applicable to the cases when a dividing surface between phases $\alpha$ and $\beta$ is used different from the surface of tension.

\section{Thermodynamic potential of a system with a sessile drop}

Neglecting gravity, the grand thermodynamic potential $\Omega$ of a system including a substrate, a one-component sessile drop and its vapour can be written as ${ }^{1}$

$$
\begin{aligned}
\Omega= & -p^{\beta} V^{\beta}-p^{\alpha} V^{\alpha}+\sigma^{\alpha \beta} A^{\alpha \beta}+\sigma^{\alpha \gamma} A^{\alpha \gamma} \\
& +\sigma^{\beta \gamma} A^{\beta \gamma}+\kappa L,
\end{aligned}
$$

where $p$ is the pressure, $V$ is the volume, $A$ is the surface area, $L$ is the length of the three-phase contact line; the single superscripts mark the quantities related to the corresponding phases.

If there is no overlapping of the liquid/substrate and liquid/vapour interfaces in the central part of the drop, the drop

\footnotetext{
${ }^{1}$ Strictly speaking, this is a hybrid function that is a grand thermodynamic potential for the liquid and vapour and free energy for the solid substrate.
}

profile will be spherical far from the three-phase contact line according to the condition of mechanical equilibrium between the liquid and the vapour in the absence of gravity. Overlapping of the surface layers is responsible for the formation of an underlying (precursor) film modifying the solid surface and leading to a difference between $\sigma^{\beta \gamma}$ and the surface tension of a "bare" solid surface. The possible presence of the precursor film on the substrate is taken into account in Eq. (2) by the term $\sigma^{\beta \gamma} A^{\beta \gamma}$. Neglecting the precursor film thickness, one can assume the entire sessile drop to have the shape of a spherical segment. Its radius and the equilibrium contact angle can be found from the condition of a minimum of the potential $\Omega$.

For the volume $V^{\alpha}$, the area $A^{\alpha \beta}$, the base radius (the radius of the three-phase contact line) $r$ and the area $A^{\alpha \gamma}$ of the base of the spherical segment with the curvature radius $R$ and the angle $\theta$ between the spherical part and the base, one can write the following expressions:

$$
\begin{aligned}
& V^{\alpha}=\frac{\pi R^{3}}{3}(2+\cos \theta)(1-\cos \theta)^{2}, \\
& A^{\alpha \beta}=2 \pi R^{2}(1-\cos \theta), \quad A^{\alpha \gamma}=\pi R^{2} \sin ^{2} \theta, \\
& r=R \sin \theta, \quad L=2 \pi R \sin \theta .
\end{aligned}
$$

For the quantities $V^{\beta}$ and $A^{\beta \gamma}$, one can write:

$$
V^{\beta}=V_{\mathrm{t}}-V^{\alpha}, \quad A^{\beta \gamma}=A_{\mathrm{t}}-A^{\alpha \gamma},
$$

where $V_{\mathrm{t}}$ is the total volume of the liquid-vapour subsystem, $A_{\mathrm{t}}$ is the total substrate area (to be more precise, the joint area of the substrate/liquid, $\alpha \gamma$, and substrate/vapour, $\beta \gamma$, surfaces).

We define the excess quantities standing in Eq. (2) by choosing the Gibbs dividing surfaces as the segment spherical surface with radius $R$ and contact angle $\theta$ and flat surfaces $\alpha \gamma$ and $\beta \gamma$ in the framework of the Gibbs method. Putting Eqs. (3)-(6) into the right-hand side of Eq. (2) yields an expression for $\Omega$ in terms of $R$ and $\theta$ :

$$
\begin{aligned}
\Omega= & -\left(p^{\alpha}-p^{\beta}\right) \frac{\pi R^{3}}{3}(2+\cos \theta)(1-\cos \theta)^{2} \\
& +2 \pi \sigma^{\alpha \beta} R^{2}(1-\cos \theta)-\left(\sigma^{\beta \gamma}-\sigma^{\alpha \gamma}\right) \pi R^{2} \sin ^{2} \theta \\
& +2 \pi \kappa R \sin \theta-p^{\beta} V_{\mathrm{t}}-\sigma^{\beta \gamma} A_{\mathrm{t}} .
\end{aligned}
$$

Evidently, potential $\Omega$ also depends on the chemical potential $\mu$ of molecules in the system and on the temperature $T$ of the system.

\section{The dependence of the line tension on the dividing surface location}

Let us see what condition follows from the variation of the location of the drop dividing surface at maintaining the physical state of the system, i.e. from the choice of various curvature radii $R$ for the drop spherical segment at 
the liquid/vapour boundary at fixed profiles of all physical characteristics within the interface. Naturally, thermodynamic potential $\Omega$ (as well as the state parameters for the bulk phases) cannot depend on the pure imaginary variation of radius $R$. Marking such variations with square brackets, we write this condition as

$\left[\frac{\mathrm{d} \Omega}{\mathrm{d} R}\right]=0$.

We first apply this condition to the case of a free drop in vapour. Then, instead of Eq. (2), we have

$\Omega=-\frac{4 \pi}{3}\left(p^{\alpha}-p^{\beta}\right) R^{3}+4 \pi \sigma^{\sigma \beta} R^{2}-p^{\beta} V_{\mathrm{t}}$

(Eq. (7) changes to Eq. (9) at $\theta=\pi$ and $A_{\mathrm{t}}=0$.) The condition (8) for Eq. (9) leads to the known Kondo equation [16]

$p^{\alpha}-p^{\beta}=\frac{2 \sigma^{\alpha \beta}}{R}+\left[\frac{\mathrm{d} \sigma^{\alpha \beta}}{\mathrm{d} R}\right]$

where the drop surface tension $\sigma^{\alpha \beta}$ is a function of $R$, whereas the pressure difference $p^{\alpha}-p^{\beta}$ plays the role of a constant. The solution of the differential Eq. (10) is

$\sigma^{\alpha \beta}=\frac{K}{R^{2}}+c R$,

where the constant $K$ is the work of the drop formation at a given value of the condensate chemical potential and $c=\left(p^{\alpha}\right.$ $\left.-p^{\beta}\right) / 3$ (these relationships can also be directly obtained from Eq. (9) taking into account that $\Omega$ is independent of $R$ ). The plot of the function (11) is characterized by a unique minimum of $\sigma^{\alpha \beta}$. The location of the dividing surface at the minimum is called surface of tension, and Eq. (10) changes to the Laplace equation

$p^{\alpha}-p^{\beta}=\frac{2 \sigma_{\mathrm{st}}^{\alpha \beta}}{R_{\mathrm{st}}}$.

In this case, we designated the radius of the surface of tension as $R_{\mathrm{St}}$ and surface tension $\sigma^{\alpha \beta}$ at $R=R_{\mathrm{st}}$ as $\sigma_{\mathrm{st}}^{\alpha \beta}$. In view of Eqs. (11) and (12), we have

$K=\frac{\sigma_{\mathrm{st}}^{\alpha \beta} R_{\mathrm{st}}^{2}}{3}, \quad c=\frac{2 \sigma_{\mathrm{st}}^{\alpha \beta}}{3 R_{\mathrm{st}}}$.

We now apply the same approach to a sessile drop in the shape of a spherical segment. We follow the scheme used in reference [7] with some adaptation for the system under consideration. Similar to the case of a free drop, all dividing surface positions should be concentric, i.e. the dividing surfaces should be conformal as defined by Gibbs [17]. However, they are segmental but not sectorial in the case of a sessile drop (Fig. 1), so that the relationships hold

$$
R \cos \theta=h=\text { const }, \quad \frac{\mathrm{d} \theta}{\mathrm{d} R}=\frac{\cot \theta}{R}, \quad \frac{\mathrm{d} r}{\mathrm{~d} R}=\frac{1}{\sin \theta} .
$$

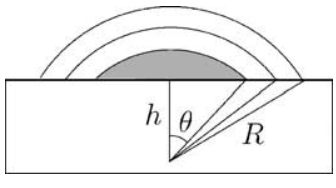

Fig. 1. The dividing surfaces and dividing lines for a spherical segment on the substrate.

Putting now Eq. (7) into Eq. (8) and taking into account Eq. (14), we obtain

$$
\begin{aligned}
{\left[\frac{\mathrm{d} \Omega}{\mathrm{d} R}\right]=} & -2 \pi R^{2}\left(p^{\alpha}-p^{\beta}\right)(1-\cos \theta)+2 \pi R \sigma^{\alpha \beta}(2-\cos \theta) \\
& +2 \pi R^{2}(1-\cos \theta)\left[\frac{\mathrm{d} \sigma^{\alpha \beta}}{\mathrm{d} R}\right]-2 \pi R\left(\sigma^{\beta \gamma}-\sigma^{\alpha \gamma}\right) \\
& +\frac{2 \pi \kappa}{\sin \theta}+2 \pi R \sin \theta\left[\frac{\mathrm{d} \kappa}{\mathrm{d} R}\right]=0 .
\end{aligned}
$$

If we now express the surface tension derivative with the aid of the Kondo equation (10), Eq. (15) immediately leads to the generalized Young equation

$\sigma^{\beta \gamma}-\sigma^{\alpha \gamma}=\sigma^{\alpha \beta} \cos \theta+\frac{\kappa}{r}+\left[\frac{\mathrm{d} \kappa}{\mathrm{d} r}\right]$.

In the right-hand side of this equation, the line tension $\kappa$ and the line tension derivative $[\mathrm{d} \kappa / \mathrm{d} r]$ with respect to the dividing surface location in the substrate plane (with respect to the location of the dividing three-phase contact line) at a fixed physical state of the system are determined at an arbitrary choice of the dividing line and the liquid/gas dividing surface.

The dependence of $\sigma^{\alpha \beta}$ and $\cos \theta$ on $r$ in Eq. (16) is determined by Eqs. (5), (10) and (14). The left-hand side of Eq. (16) is given by the difference $\sigma^{\beta \gamma}-\sigma^{\alpha \gamma}$, which is constant at a fixed physical state of the system. Recognizing this, we rewrite (16) as a differential equation

$\left[\frac{\mathrm{d} \kappa}{\mathrm{d} r}\right]+\frac{\kappa}{r}+\frac{a}{\left(r^{2}+h^{2}\right)^{3 / 2}}=b$,

where we have introduced the notations

$a \equiv K h, \quad b \equiv \sigma^{\beta \gamma}-\sigma^{\alpha \gamma}-c h$.

Thus, taking into account the said above, three constants $h, a$ and $b$ are present in Eq. (17).

The solution of Eq. (17) is

$\kappa=\frac{b r}{2}+\frac{a}{r\left(r^{2}+h^{2}\right)^{1 / 2}}+\frac{d}{r}$,

where the integration constant $d$ can be expressed through the radius $r=r_{\text {lt }}$ of the dividing surface on the substrate plane at the extreme point for the line tension $\kappa$ as a function of $r$ :

$d=\frac{b r_{\mathrm{lt}}^{2}}{2}+\frac{a\left(2 r_{\mathrm{lt}}^{2}+h^{2}\right)}{\left(r_{\mathrm{lt}}^{2}+h^{2}\right)^{3 / 2}}$.

Equation can also be directly obtained from Eq. (7) by expressing $\Omega$ in terms of $r$ and $h$ and using Eq. (11). 
By analogy with the surface of tension, the dividing line characterized by $r=r_{\mathrm{lt}}$ and for which $[\mathrm{d} \kappa / \mathrm{d} r]=0$ can be called the line of tension [7]. With this choice of a dividing surface, the generalized Young equation (16) takes the simplest form

$\sigma^{\beta \gamma}-\sigma^{\alpha \gamma}=\sigma^{\alpha \beta} \cos \theta+\frac{\kappa_{\mathrm{lt}}}{r_{\mathrm{lt}}}$,

where $\kappa_{\mathrm{lt}}=\left.\kappa\right|_{r=r_{\mathrm{lt}}}$.

As it follows from Eq. (17), the value $\kappa_{\mathrm{lt}}$ of the line tension can be expressed through the parameters $h, a$ and $b$ and the radius $r_{\mathrm{lt}}$ as

$\kappa_{\mathrm{lt}}=b r_{\mathrm{lt}}-\frac{a r_{\mathrm{lt}}}{\left(r_{\mathrm{lt}}^{2}+h^{2}\right)^{3 / 2}}$.

According to the definitions (18) and (13), the constant $a$ is positive, whereas the constant $b$ can be either positive or negative depending on the substrate wettability. Since, obviously, $r_{\mathrm{lt}}>0$, the value of $\kappa_{\mathrm{lt}}$ can be either positive (at $b>a /\left(r_{\mathrm{lt}}^{2}+h^{2}\right)^{3 / 2}$ ) or negative (at $\left.b<a /\left(r_{\mathrm{lt}}^{2}+h^{2}\right)^{3 / 2}\right)$.

It is convenient to pass to dimensionless quantities in Eq. (19), $\tilde{\kappa} \equiv \kappa / \kappa_{\mathrm{lt}}$ and $\tilde{r} \equiv r / r_{\mathrm{lt}}$, using Eq. (20) and excluding constant $b$ with the aid of Eq. (22).

As a result, we obtain, instead of Eq. (19),

$$
\begin{aligned}
\tilde{\kappa}= & \frac{1}{2}\left(\tilde{r}+\frac{1}{\tilde{r}}\right) \\
& +A\left[\tilde{r}+\frac{2}{\tilde{r} \sin ^{3} \theta_{\mathrm{lt}}\left(\tilde{r}^{2}+\cot ^{2} \theta_{\mathrm{lt}}\right)^{1 / 2}}-\frac{3+2 \cot ^{2} \theta_{\mathrm{lt}}}{\tilde{r}}\right],
\end{aligned}
$$

where only two parameters remain, $A$ and $\theta_{\mathrm{lt}}$,

$$
A \equiv \frac{a r_{\mathrm{lt}}}{2 \kappa_{\mathrm{lt}}\left(r_{\mathrm{lt}}^{2}+h^{2}\right)^{3 / 2}}, \quad \theta_{\mathrm{lt}} \equiv \operatorname{arccot} \frac{h}{r_{\mathrm{lt}}} \text {. }
$$

These parameters can be regarded as independent, with the sign of parameter $A$ being determined by the sign of the line tension $\kappa_{\mathrm{lt}}$.

Fig. 2 shows the dependence of the dimensionless line tension $\tilde{\kappa}$ on the dimensionless radius $\tilde{r}$ of a dividing line at $\theta_{\mathrm{lt}}$ $=0.1$ and at three values of parameter $A: A=0.5, A=-0.1$ and

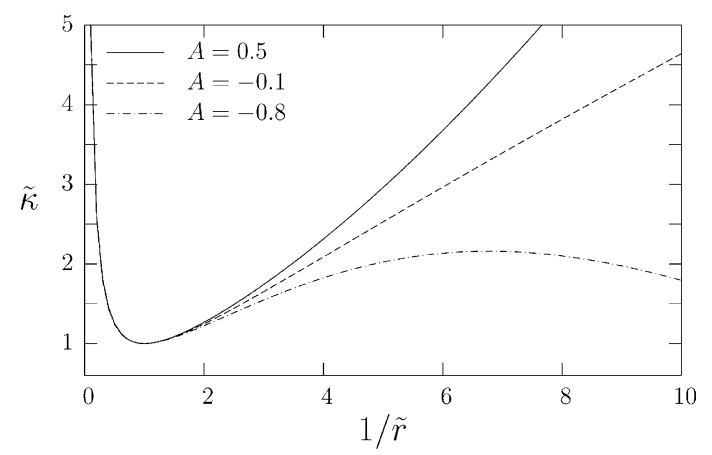

Fig. 2. The dependence of the line tension $\tilde{\kappa}$ on the radius $\tilde{r}$ of a dividing line at $\theta_{\mathrm{lt}}=0.1$ and at three different values of parameter $A: A=0.5, A=-0.1$ and $A=-0.8$.
$A=-0.8$. In all cases considered, the value $\tilde{\kappa}=1$ corresponds to the local minimum in the curve $\tilde{\kappa}(\tilde{r})$. Because of Eqs. (22) and (24), the absolute values of $A$ must be smaller than unity (even smaller than $1 / 2$ at negative $b$ and $\kappa_{\mathrm{lt}}$ ). Therefore, the appearance of a maximum in the case of negative line tensions in Fig. 2 at $A=-0.8$ can be an artefact.

We designate as $r_{\mathrm{st}}$ the radius of the dividing line, on the substrate, corresponding to the choice of the surface of tension as a dividing surface between the liquid and the vapour. From the above phenomenological analysis, it is hard to deduce how the quantities $r_{\mathrm{st}}$ and $r_{\mathrm{lt}}$ are related to each other. One can only expect that their coincidence can be completely random and rare. In particular, this means that there is no reason for neglecting the last term in Eq. (16) when using the surface of tension (as well as the equimolecular surface) as a dividing surface between the liquid and the vapour.

\section{The equilibrium condition at the three-phase contact line}

We now return to Eq. (7) for the thermodynamic potential $\Omega$ of the system to derive the condition of equilibrium at the three-phase contact line. Applying the equilibrium principle to $\Omega$, we have

$$
\left(\frac{\partial \Omega}{\partial \theta}\right)_{T, \mu, R}=0
$$

The location of a dividing surface is now considered as given at fixed conditions (and is arbitrary, since the conditions themselves are arbitrary). Fixing the temperature and chemical potential means the constancy of all state parameters of the system including the curvature radius $R$ of the drop surface. By contrast, the distance $h$ (see Fig. 1) becomes variable. Herewith the relationship is fulfilled

$$
\left(\frac{\partial r}{\partial \theta}\right)_{T, \mu, R}=R \cos \theta
$$

Putting Eq. (7) into Eq. (25) taking into account Eq. (26) yields

$$
\begin{aligned}
\left(\frac{\partial \Omega}{\partial \theta}\right)_{T, \mu, R}= & -\left(p^{\alpha}-p^{\beta}\right) \pi R^{3} \sin ^{3} \theta+2 \pi \sigma^{\alpha \beta} R^{2} \sin \theta \\
& +2 \pi \kappa R \cos \theta-2 \pi\left(\sigma^{\beta \gamma}-\sigma^{\alpha \gamma}\right) R^{2} \sin \theta \cos \theta \\
& +2 \pi R^{2} \sin \theta \cos \theta\left(\frac{\partial \kappa}{\partial r}\right)_{T, \mu}=0
\end{aligned}
$$

It is implied here that the line tension $\kappa=\kappa(T, \mu, r)$ does not explicitly depend on the contact angle $\theta$. If we now replace the difference $p^{\alpha}-p^{\beta}$ by the expression following from the Kondo equation (10), Eq. (27) changes to the generalized Young equation at a certain given (by external conditions) location of the dividing surface and at a dividing line corre- 
sponding to this dividing surface:

$$
\sigma^{\beta \gamma}-\sigma^{\alpha \gamma}=\sigma^{\alpha \beta} \cos \theta+\frac{\kappa}{r}+\left(\frac{\partial \kappa}{\partial r}\right)_{T, \mu}-\frac{r \tan \theta}{2}\left[\frac{\mathrm{d} \sigma^{\alpha \beta}}{\mathrm{d} R}\right]_{(28)}
$$

Taking the surface of tension as the dividing surface, Eq. (28) becomes

$\sigma^{\beta \gamma}-\sigma^{\alpha \gamma}=\sigma^{\alpha \beta} \cos \theta+\frac{\kappa}{r}+\left(\frac{\partial \kappa}{\partial r}\right)_{T, \mu}$,

which coincides with Eq. (1) at $\varphi=0$. Comparing the righthand sides of Eqs. (16) and (29), we arrive at the relationship

$$
\left(\frac{\partial \kappa}{\partial r}\right)_{T, \mu}=\left[\frac{\mathrm{d} \kappa}{\mathrm{d} r}\right]+\frac{r \tan \theta}{2}\left[\frac{\mathrm{d} \sigma^{\alpha \beta}}{\mathrm{d} R}\right] .
$$

This equation relates the physical dependence of the line tension on the radius of the three-phase contact line at given temperature and chemical potential to the imaginary dependence of the line and surface tensions on the dividing surface location. If the surface of tension is chosen as a dividing surface, the surface tension derivative in Eq. (30) becomes zero, and we arrive at a remarkable relationship

$$
\left(\frac{\partial \kappa}{\partial r}\right)_{T, \mu}=\left[\frac{\mathrm{d} \kappa}{\mathrm{d} r}\right]
$$

Eq. (31) means that, with this choice of a dividing surface, the derivative of the line tension with respect to radius of the three-phase contact line at constant temperature and chemical potential coincides with the line tension derivative at an imaginary shift of the dividing surface at fixed physical state of the system.

\section{The role of linear adsorption}

As it was shown above, there remains a possibility for the line tension variation together with the radius of the threephase contact line even when a certain choice is made for the location of the dividing surface between the liquid and the vapour and all the state parameters of the bulk phases and surfaces, like the temperature and the chemical potential, are fixed. The corresponding derivative stands in the equilibrium condition expressed by Eq. (28).

To write the expression for the total differential of the line tension, it is sufficient to supplement the partial differential corresponding to this derivative with the terms with differentials of temperature and chemical potential:

$\mathrm{d} \kappa=\left(\frac{\partial \kappa}{\partial r}\right)_{T, \mu} \mathrm{d} r-s_{1} \mathrm{~d} T-\Lambda \mathrm{d} \mu$.

Coefficients $s_{1}$ and $\Lambda$ are known as the linear excesses of entropy and matter, respectively, related to the unit length of the three-phase contact line. Eq. (32) itself is nothing else as the linear adsorption equation $[15,18]$ called by analogy with the
Gibbs adsorption equation for surfaces (the excess $\Lambda$ plays the role of a linear adsorption). At a constant temperature, Eq. (32) yields an important relationship

$$
\left(\frac{\partial \kappa}{\partial r}\right)_{T, \mu}=\frac{\mathrm{d} \kappa}{\mathrm{d} r}+\Lambda \frac{\mathrm{d} \mu}{\mathrm{d} r}
$$

In particular, Eq. (33) permits us finding the partial derivative $(\partial \kappa / \partial r)_{T, \mu}$ from the linear adsorption value $\Lambda$ and the derivatives $\mathrm{d} \kappa / \mathrm{d} r$ and $\mathrm{d} \mu / \mathrm{d} r$ along equilibrium states. For example, such calculations are possible by applying the density functional method based on the models for intermolecular potentials $[10,11,20]$ and by applying the method of the functional of the local thickness of a liquid film based on the approximations for the isotherm of the disjoining pressure as a function of the film thickness $[8,9,11]$. Both these methods are capable of independent calculating the contact angle, the chemical potential of molecules in a system and the linear adsorption as functions of the dividing line radius.

Some assertions can be made without using any specific model. Let us assume that the line tension $\kappa$ and the linear adsorption $\Lambda$ expressed as functions of the equilibrium contact line radius $r$ have the following asymptotic behavior:

$\kappa=\kappa_{0}+O\left(r^{-1}\right), \quad \Lambda=\Lambda_{0}+O\left(r^{-1}\right)$

with the finite limits $\kappa_{0}$ and $\Lambda_{0}$ corresponding to the bulk coexistence at $\mu \rightarrow \mu_{0}$ (where $r \rightarrow \infty$, i.e. the contact line becomes straight). Under assumption of incompressible liquid, we have for the pressure difference

$p^{\alpha}-p^{\beta} \approx \frac{\mu-\mu_{0}}{v^{\alpha}}$

with $v^{\alpha}$ the molecular volume in $\alpha$ phase. Using the Laplace equation (12) and Eq. (34), one can derive $\mathrm{d} \mu / \mathrm{d} r=$ $-2 \sigma^{\alpha \beta} v^{\alpha} r^{-2} \sin \theta$. It leads to the following estimation:

$\Lambda \frac{\mathrm{d} \mu}{\mathrm{d} r}=-2 \Lambda_{0} \sigma_{0}^{\alpha \beta} v^{\alpha} r^{-2} \sin \theta_{0}+O\left(r^{-3}\right)$

where $\sigma_{0}^{\alpha \beta}$ is the value of the surface tension $\sigma^{\alpha \beta}$ at $\mu=\mu_{0}$, $\theta_{0}$ is the limit macroscopic value of the contact angle. According to Eq. (34), one can estimate the derivative $\mathrm{d} \kappa / \mathrm{d} r$ as $\mathrm{d} \kappa / \mathrm{d} r=O\left(r^{-2}\right)$. It means that under the assumptions (34), the partial derivative $(\partial \kappa / \partial r)_{T, \mu}=O\left(r^{-2}\right)$, while for the term $\kappa / r$ on the right-hand side of the generalized Young equation (29), the estimation $\kappa / r=\kappa_{0} / r+O\left(r^{-2}\right)$ can be made. Therefore, if the linear adsorption $\Lambda$ has a finite limit at bulk phase coexistence, the term $(\partial \kappa / \partial r)_{T, \mu}$ can be neglected for sufficiently large droplets and the term $\kappa / r$ can be approximated with its asymptotic form $\kappa_{0} / r$.

\section{Conclusion}

The analysis presented ascertains the role of the term with the line tension derivative with respect to the radius of the three-phase contact line when using the generalized Young 
equation for establishing relations between the contact angle, the line tension and the radius of the three-phase contact line. The generalized Young equation with $\kappa=$ const and, correspondingly, without the term $\partial \kappa / \partial r$ (see, e.g., [2], and also references in reference [19]) has repeatedly been discussed in the literature. In other papers, the dependence $\kappa(r)$ has been granted in the generalized Young equation, but the contribution of the partial derivative $\partial \kappa / \partial r$ [21] has been neglected. In the third case, the partial derivative $\partial \kappa / \partial r$ in the generalized Young equation had been replaced by the total derivative $\mathrm{d} \kappa / \mathrm{d} r$ along the equilibrium states (as, for example, it was actually made in reference [3]) and, in this way, the linear adsorption (see Eq. (33)) had been neglected.

In the limit of large drops, when $\mu$ tends to the chemical potential $\mu_{0}$ for coexisting bulk phases $\alpha$ and $\beta$, the terms containing the line tension in Eq. (29) become negligible. Then, the equation changes to the classical Young equation

$\sigma_{0}^{\alpha \beta} \cos \theta_{0}=\sigma_{0}^{\beta \gamma}-\sigma_{0}^{\alpha \gamma}$,

where $\sigma_{0}^{\alpha \beta}, \sigma_{0}^{\beta \gamma}$ and $\sigma_{0}^{\alpha \gamma}$ are the corresponding surface tensions at $\mu=\mu_{0}$.

Comparing the generalized Young equation (29) with the classical one Eq. (36), variations of surface tensions $\sigma^{\alpha \beta}, \sigma^{\alpha \gamma}$ and $\sigma^{\beta \gamma}$ related to the variation of chemical potential $\mu$ of the whole system are often neglected to write

$\cos \theta_{0}-\cos \theta=\frac{1}{\sigma_{0}^{\alpha \beta}}\left(\frac{\kappa}{r}+\frac{\partial \kappa}{\partial r}\right)$.

In this way, the adsorptions $\Gamma^{\alpha \beta}, \Gamma^{\alpha \gamma}$ and $\Gamma^{\beta \gamma}$ at the corresponding interfaces are evidently neglected, or the equimolecular surface with respect to the fluid component is chosen as the dividing surface. However, if such choice has been made for the dividing surfaces $\alpha \gamma$ and $\beta \gamma$, it is necessary to include into consideration the chemical potential of the component of a solid substrate. This significantly complicates the analysis, so that it is more natural to choose the corresponding dividing surfaces equimolecular with respect to the solid component. Choosing the equimolecular surface as the dividing surface $\alpha \beta$, adsorption $\Gamma^{\alpha \beta}$ becomes exactly equal to zero. In this case, however, Eqs. (10) and (28) expressing the equilibrium condition become different from the Laplace equation (12) and the generalized Young equation (29).

Whereas Eq. (29) derived within phenomenological thermodynamics is rigorous at the choice of dividing surfaces as is indicated, its various truncated forms can be used, if at all, only in restricted regions.

\section{References}

[1] A.I. Rusanov, Colloid J. USSR 39 (1977) 618.

[2] B.V. Toshev, M.Z. Avramov, Colloids Surf. A 75 (1993) 33.

[3] N.V. Churaev, V.M. Starov, B.V. Derjaguin, J. Colloid Interface Sci. 89 (1982) 16.

[4] A.I. Rusanov, Kolloid Zh. 60 (1998) 815.

[5] A.I. Rusanov, Mendeleev Commun. No. 1 (1996) 30.

[6] L. Boruvka, A.W. Neumann, J. Chem. Phys. 66 (1977) 5464.

[7] A.I. Rusanov, Colloid J. USSR 49 (1987) 614.

[8] J.O. Indekeu, Physica A 183 (1992) 439.

[9] Yu. Solomentsev, L.R. White, J. Colloid Interface Sci. 218 (1999) 122.

[10] T. Getta, S. Dietrich, Phys. Rev. E 57 (1998) 655.

[11] C. Bauer, S. Dietrich, Eur. Phys. J. B 10 (1999) 767.

[12] V.G. Babak, Colloids Surf. A 156 (1999) 423; V.G. Babak, Rev. Chem. Eng. 15 (1999) 157.

[13] D. Li, Colloids Surf. A 116 (1996) 1.

[14] P. Seppecher, Oil Gas Sci. Technol. - Revue de l'Institut Français du Pétrole 56 (2001) 77.

[15] J.S. Rowlinson, B. Widom, Molecular Theory of Capillarity, Clarendon Press, Oxford, 1982.

[16] S. Kondo, J. Phys. Soc. Jpn. 10 (1955) 381.

[17] J.W. Gibbs, Trans. Conn. Acad. 3 (1878) 343.

[18] P. Chen, Colloids Surf. A 161 (2000) 23.

[19] A.I. Rusanov, Colloids Surf. A 156 (1999) 315.

[20] V. Talanquer, D.W. Oxtoby, J. Chem. Phys. 104 (1996) 1483.

[21] B.V. Derjaguin, N.V. Churaev, V.M. Muller, Surface Forces, Consultants Bureau, Plenum Press, New York, 1987. 